\title{
Effect of enamel protective agents on shear bond strength of orthodontic brackets
}

\author{
Mona A Montasser ${ }^{*}$ and Mahasen Taha
}

\begin{abstract}
Background: This paper aimed to study the effect of two enamel protective agents on the shear bond strength (SBS) of orthodontic brackets bonded with conventional and self-etching primer (SEP) adhesive systems.

Methods: The two protective agents used were resin infiltrate (ICON) and Clinpro; the two adhesive systems used were self-etching primer system (Transbond Plus Self Etching Primer + Transbond XT adhesive) and a conventional adhesive system (37\% phosphoric acid etch + Transbond XT primer + Transbond XT adhesive ). Sixty premolars divided into three major groups and six subgroups were included. The shear bond strength was tested $72 \mathrm{~h}$ after bracket bonding. Adhesive remnant index scores (ARI) were assessed. Statistical analysis consisted of a one-way ANOVA for the SBS and Kruskal-Wallis test followed by Mann-Whitney test for the ARI scores.
\end{abstract}

Results: In the control group, the mean SBS when using the conventional adhesive was 21.1 $\pm 7.5 \mathrm{MPa}$ while when using SEP was $20.2 \pm 4.0 \mathrm{MPa}$. When ICON was used with the conventional adhesive system, the SBS was $20.2 \pm$ 5.6 MPa while with SEP was $17.6 \pm 4.1 \mathrm{MPa}$. When Clinpro was used with the conventional adhesive system, the SBS was $24.3 \pm 7.6 \mathrm{MPa}$ while with SEP was 11.2 $\pm 3.5 \mathrm{MPa}$. Significant differences in the shear bond strength of the different groups $(P=.000)$ was found as well as in the ARI scores distribution $(P=.000)$.

Conclusion: The type of the adhesive system used to bond the orthodontic brackets, either conventional or self-etching primer, influenced the SBS, while the enamel protective material influenced the adhesive remnant on the enamel surface after debonding.

Keywords: Enamel protective agents; Shear bond strength; ARI scores

\section{Background}

Enamel demineralization and white spot lesions associated with orthodontic fixed appliances is one of the greatest challenges faced by clinicians at the end of the orthodontic treatment not only for esthetic reasons but also because this subsurface demineralization represents the first stage of caries formation [1-4].

Different methods have been studied, all aiming to reduce enamel demineralization during orthodontic treatment without compromising the bond strength of the orthodontic brackets. The most common method was the use of fluoride-containing mouth rinses, gels, and tooth pastes [5-7]; however, studies found a significant association between the patient compliance to the rinsing program advised by the clinician and the reduction

\footnotetext{
* Correspondence: mmontasser11@yahoo.com

Department of Orthodontics, Faculty of Dentistry, Mansoura University, Mansoura 35516, Egypt
}

\section{Springer}

(c) 2014 Montasser \& Taha; licensee Springer. This is an Open Access article distributed under the terms of the Creative Commons Attribution License (http://creativecommons.org/licenses/by/2.0), which permits unrestricted use, distribution, and reproduction in any medium, provided the original work is properly cited. in the development of white spot lesions [8]. It was found that with only standardized general prophylactic measures, new white spot lesions developing on the maxillary front teeth during orthodontic treatment were seen in $60.9 \%$ of the patients [9].

Preventive measures that do not depend on the patient's compliance have been developed and gained popularity to solve the problem of demineralization. These included the use of glass ionomer cement $[10,11]$, topical applications of preventive agents as fluoride and casein phosphopeptide-amorphous calcium phosphate $[12,13]$, antibacterial agents incorporated in the adhesive resin $[14,15]$, fluoride releasing adhesives [16,17], caries infiltration resins $[18,19]$, laser irradiation $[20,21]$, bioactive glass-containing adhesives [22], and enamel deproteinizing agents [23].

The current study focused on two preventive agents Clinpro and ICON. Clinpro is a fluoridated varnish 
containing $5 \%$ sodium fluoride. Fluoride was found to be effective in reducing the development of white spot lesions associated with fixed orthodontic treatment $[16,24]$. Also, ICON resin infiltration was found to decrease the dissolution of enamel and so limit the appearance of white spot lesions [25]. When is the proper timing to apply these materials to get the best result of decreasing the white spot lesions around the orthodontic brackets is a worthwhile question. These preventive agents could be applied after bonding the orthodontic brackets, but this may not be easy all the times especially where there are severely crowded or partially erupted teeth. The other option is to apply these materials before bonding the orthodontic brackets, but these preventive agents could have an effect on the shear bond strength and/or the amount of adhesive left on the teeth after debonding of the orthodontic brackets upon treatment completion.

The objective of this study was to study the effect of using the two enamel protective agents before bonding on the shear bond strength of orthodontic brackets bonded with conventional and self-etch adhesive systems.

\section{Methods}

This in vitro testing used 60 extracted human upper premolars stored in an aqueous solution of thymol $(0.1 \%$ $\mathrm{wt} / \mathrm{vol})$. Teeth were extracted as part of orthodontic treatment and collected to be used in research. To calculate the sample size, Epicalc 2000 software version 1.02 (Brixton Books, Brixton, UK) was used. The sample size was found to be ten specimens for each group based on $80 \%$ power and $95 \%$ confidence interval.

The teeth were fixed in self-curing acrylic resin placed in flexible molds with the roots embedded in the acrylic and the crown exposed and oriented perpendicularly to the bottom of the mold.

Two types of enamel protective agents were used in the current study: ICON (DMG, Hamburg, Germany) and Clinpro (3M Unitek, Monrovia, CA, USA). The two adhesive systems used in this study were Transbond XT light cure adhesive and Transbond Plus Self Etching Primer (3M Unitek, Monrovia, CA, USA), and Transbond XT light cure adhesive, Transbond XT primer, and 37\% phosphoric acid (3M Unitek, Monrovia, CA, USA). All materials were used according to the manufacturers' instructions.

The sample was divided into three major groups; group 1 used (ICON) before bonding the orthodontic brackets, group 2 used Clinpro before bonding, and group 3 a control group with no protective enamel agent used. Each group was divided into two subgroups; in the first one, orthodontic brackets were bonded with self-etching adhesive system and in the second one, a conventional adhesive system was used.
Premolar stainless steel brackets (Equilibrium 2 Roth prescription, 0.022 in. slot size, Dentaurum Orthodontics, Ispringen, Germany) were used. The buccal surface of each tooth was cleaned with non-fluoride oil-free pumice paste using a nylon brush attached to a slow-speed hand piece for $5 \mathrm{~s}$, and then the tooth was rinsed with water for $10 \mathrm{~s}$ and dried with an oil-free air spray. Brackets were bonded to the teeth according to the manufacturer's instructions for the adhesive system and stored in distilled water at $37^{\circ} \mathrm{C}$ until testing.

Bracket debonding was performed $72 \mathrm{~h}$ after bonding in a material testing unit (model no 5500, Instron Corp, Canton, MA, USA) with an occluso-gingival load applied to the bracket base. The shearing rod was adjusted each time so the shearing blade is parallel to the base of the bracket contacting it in a reproducible way each test. The shear force was applied to the bracket by lowering the shearing rod perpendicularly in the gingival direction, producing a shear force at the bracket-enamel interface (Figure 1).

The crosshead speed was $2.0 \mathrm{~mm} / \mathrm{min}$, and the failure load in Newton was divided by the bracket base bonding area of $10.90 \mathrm{~mm}^{2}$ to calculate the shear bonding strength in $\mathrm{MPa}$.

The adhesive remnant index (ARI) and failure site assessment was completed immediately after each shear bond strength debonding under $\times 20$ magnification [26]. The ARI evaluation used the 4-point scale of Artun and Bergland [27] where 0 indicates no adhesive left on the tooth surface, implying that bond fracture occurred at

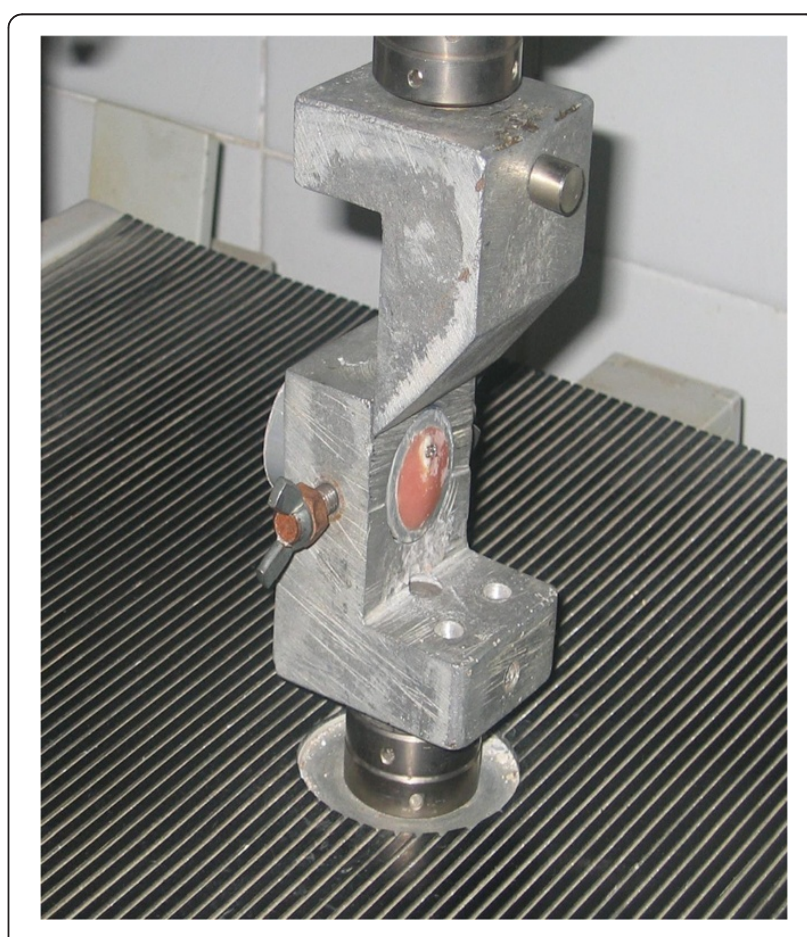

Figure 1 Typically mounted specimen for SBS testing. 
the resin/enamel interface; 1 indicates less than half the resin left on the tooth surface, implying that bond fracture occurred predominantly at the resin/enamel interface; 2 indicates more than half the resin left on the tooth surface, implying that bond fracture occurred predominantly at the bracket/resin interface; and 3 indicates all resin left on the tooth surface, with a distinct impression of the bracket base, implying that bond fracture occurred at the bracket/resin interface.

Descriptive statistics, including mean, standard deviation, and minimum and maximum values of the shear bond strength, were calculated for each of the adhesive systems tested. Analysis of variance (ANOVA) test followed by a LSD post hoc multimeans comparison test was used to compare the groups. A Kruskal-Wallis test was used in conjunction with a Mann-Whitney test to compare the differences in the ARI scores between the groups. Significance for all statistical tests was at $P \leq .05$. Statistics were carried out using Statistical Package for Social Sciences (SPSS Inc, Chicago, IL, USA) program version 10 .

\section{Results}

The descriptive statistics of the SBS of each group are presented in Table 1. The one-way ANOVA, Table 1, indicated significant differences in the shear bond strength of the different groups $(P=.000)$. When using Clinpro before bonding with SEP and Transbond XT, the SBS was significantly less than the other groups; the two control groups, the conventional adhesive group $(P=.000)$ and the SEP group $(P=.001)$; the two ICON groups, the conventional adhesive $(P=.001)$ and the SEP group $(P=.015)$; and Clinpro with the conventional adhesive system $(P=.000)$. When using Clinpro before bonding with the conventional adhesive, the bond strength was similar to that of the other groups but significantly higher than the SBS when using ICON before bonding with SEP and Transbond XT adhesive.

The results of the Kruskal-Wallis test showed that the ARI scores, Table 2 , were significantly different $(P=.000)$ between the groups. The Mann-Whitney test showed no difference in the ARI scores between self-etching and conventional etching groups when using ICON $(P=.166)$,
Table 2 Frequencies of the ARI scores for the two groups

\begin{tabular}{llllll}
\hline & Number & \multicolumn{5}{c}{ ARI scores } \\
& & $\mathbf{0}$ & $\mathbf{1}$ & $\mathbf{2}$ & $\mathbf{3}$ \\
\hline Transbond XT + ICON + $\mathrm{H}_{3} \mathrm{PO}_{4}{ }^{a}$ & 10 & 0 & 1 & 1 & 8 \\
Transbond XT + ICON + $\mathrm{SEP}^{a}$ & 10 & 0 & 3 & 2 & 5 \\
Transbond XT + Clinpro $+\mathrm{H}_{3} \mathrm{PO}_{4}{ }^{\mathrm{b}}$ & 10 & 5 & 3 & 2 & 0 \\
Transbond XT + Clinpro + $\mathrm{SEP}^{\mathrm{b}}$ & 10 & 6 & 2 & 1 & 1 \\
Transbond XT + primer $+\mathrm{H}_{3} \mathrm{PO}_{4}{ }^{c}$ & 10 & 2 & 4 & 2 & 2 \\
Transbond XT + SEP & 10 & 3 & 4 & 0 & 3 \\
\hline
\end{tabular}

Chi-square $=22.77 ; P=.000 ; 0$ indicates no adhesive left on the tooth surface, 1 indicates less than half the resin left on the tooth surface, 2 indicates more than half the resin left on the tooth surface, and 3 indicates all resin left on the tooth surface, with a distinct impression of the bracket base. Scores in each row with the same letter are not significantly different at $P \leq .05$.

Clinpro $(P=.802)$, as well as when bonding to untreated enamel in the control group $(P=.751)$. Results of one-way ANOVA showed also that the type of preventive agent used on the enamel significantly influenced the ARI scores distribution; there was a significant difference depending on whether it was ICON or Clinpro that was used before bonding with SEP $(P=.005)$ or with phosphoric acid etching $(P=.000)$.

\section{Discussion}

The lowest SBS was recorded with the samples treated with Clinpro before bonding the orthodontic brackets; the SBS in this group was significantly lower than the SBS in the other five groups. This could be attributed to the resistance effect that the outer enamel layer acquires from the fluoride content of the Clinpro which may be of significant effect especially when using self-etching primers in bonding due to their more superficial etching effect compared with the etching of the conventionally used phosphoric acid. Previous studies [28-30] with scanning electron microscope (SEM) indicated that although selfetch priming agents have the potential to etch the enamel surface, the etching pattern is less deep compared to the etching pattern of phosphoric acid. A chemical bonding capacity through the interaction between some functional monomers and the calcium of residual hydroxyapatite may contribute favorably to the bonding effectiveness [31-33], but fluoride affects the enamel surface rendering it more

Table 1 Descriptive statistics of the in vitro shear bond strength (MPa)

\begin{tabular}{|c|c|c|c|c|c|}
\hline & Number & Mean & SD & Minimum & Maximum \\
\hline Transbond XT + ICON + $\mathrm{H}_{3} \mathrm{PO}_{4}{ }^{\mathrm{a}, \mathrm{d}}$ & 10 & 20.2 & \pm 4.6 & 11.0 & 28.6 \\
\hline Transbond XT + ICON + SEP bd & 10 & 17.6 & \pm 4.1 & 13.3 & 23.7 \\
\hline Transbond XT + Clinpro $+\mathrm{H}_{3} \mathrm{PO}_{4}{ }^{\mathrm{a}}$ & 10 & 21.3 & \pm 6.6 & 13.4 & 34.2 \\
\hline Transbond XT + Clinpro + SEP ${ }^{c}$ & 10 & 11.2 & \pm 3.5 & 5.6 & 15.0 \\
\hline Transbond XT + primer $+\mathrm{H}_{3} \mathrm{PO}_{4}{ }^{\mathrm{a}, \mathrm{d}}$ & 10 & 21.1 & \pm 7.5 & 11.2 & 34.7 \\
\hline Transbond XT + SEP ${ }^{\mathrm{a}, \mathrm{d}}$ & 10 & 20.2 & \pm 4.0 & 14.0 & 25.9 \\
\hline
\end{tabular}

$F=6.07 ; P=.000$. Mean values in each row with the same letter are not significantly different at $P \leq .05$. 
resistant to demineralization. Fluoride in low concentrations favors the formation of fluoro-hydroxyapatite, which is less susceptible to acidic solubility than hydroxyapatite $[34,35]$. Therefore, it is recommended to use these preventive agents after bonding the brackets when self-etch adhesive systems are used.

In the current study, using the caries infiltrant (ICON) before bonding did not significantly change the bond strength compared to the other groups, although the bond strength was lower when self-etching primer was used than when phosphoric acid was used for enamel preparation before bonding. This was also observed in the control group; shear bond strength was lower when self-etching primer was used than when phosphoric acid was used, but this difference was statistically insignificant. Previous studies found a significant increase in the shear bond strength of Transbond XT adhesive with phosphoric acid and Transbond XT primer when ICON was used before bonding orthodontic brackets to sound enamel [36] or even to demineralized enamel [37]. The shear bond strength was also increased when Transbond Plus Self Etching Primer was used instead of the conventional phosphoric acid etching to sound enamel [36]. The shear bond strengths recorded in this study were sufficient for clinical use in all the six groups presenting different combinations of adhesive systems and enamel protective agents as well as control groups. The average range of bond strength was suggested by Reynolds [38] to be 5.9 to $7.8 \mathrm{MPa}$ for clinical and $4.9 \mathrm{MPa}$ for laboratory performances. In vitro and in vivo studies of SBS are both needed; in vitro measurements of shear bond strength provide useful information about the bonding efficiency of different types of materials, but the actual performance of these materials can only be evaluated in the environment where they were intended to function [39]. Unfortunately, no one variable or combination of variables that can be measured in the laboratory is perfectly predictive of what might occur when the bonding adhesive is used in the demanding environment of the oral cavity [40-42]. Therefore; in vitro studies are mainly important as a preliminary guide to the clinician, while in vivo studies are needed for evidence-based practice.

The distribution of the ARI scores was assessed in this study under $\times 20$ magnifications [26]. Although different quantitative and qualitative methods have been used to assess the ARI scores after orthodontic bracket debonding and quantitative methods were found preferable if accurate evaluation of the adhesive remnant is required [43], ARI score evaluation system has proved to be of value in the studies of orthodontic adhesive systems. ARI score system is a quick and simple method that needs no special equipment. Although SEM evaluation might be more accurate than evaluation under $\times 10$ or $\times 20$ magnification, it is harder to be reflected in clinical applications [26]. The distribution of the ARI scores was found different between the three major groups. In the ICON group, for both selfetching and conventional etching subgroups, higher ARI scores tended to be more frequent, while in Clinpro and control groups, both self-etching and conventional etching subgroups, less adhesive remnant tended to be seen left on the enamel surface after debonding. This could be attributed to the chemical bond between the resin infiltrant and the adhesive resin. However, the adhesive remaining on the enamel surface after debonding was not different in the three major groups between the self-etching subgroup and the conventional etching subgroup indicating a similar effect of the enamel protective material with the two types of adhesive systems. These results differed from the results of Naidu et al. [36] study that found using ICON as preconditioning before bonding orthodontic brackets to sound enamel did not affect ARI scores distribution compared to the control groups using Transbond $\mathrm{XT}$ primer and Transbond PSEP. The importance of the site of bond failure was found not to be a reflection of bond strength; therefore, the site of failure did not reflect different bond strengths at different interfaces $[44,45]$. On the other hand, a variety of factors could affect bond strength including the type of enamel conditioner, acid concentration, length of etching time, composition of the adhesive, bracket base design, bracket material, oral environment, skill of the clinician, and time of light exposure in case of light-cure approach [46].

Applying the results of this study clinically, it would be preferred using Clinpro after bonding the orthodontic brackets when self-etch adhesive systems are used, while it could be used before bonding when conventional adhesive systems are used. ICON resin infiltrate, on the other hand, could be used before bonding with either of the two adhesive systems, but removal of large amount of adhesive remnant would be needed.

\section{Conclusions}

Based on the above findings, we conclude the following:

1. Overall, the SBS was lower when self-etching primer was used than when phosphoric acid was used for enamel preparation before bonding in the three major groups.

2. Significantly lower SBS was recorded when Clinpro was used before bonding using the self-etching adhesive system.

3. The ICON group showed the higher ARI scores to be more frequent, while Clinpro and control groups showed lower ARI scores more frequently. The adhesive remnant was not different between the self-etching and the conventional etching subgroups. 


\section{Competing interests}

The authors declare that they have no competing interests.

\section{Authors' contributions}

MM formulated the idea and participated in designing the study, participated in experimental part (sample preparation, orthodontic bracket bonding, and testing procedure), and wrote the manuscript. MT participated in designing the study, participated in experimental part (sample preparation and orthodontic bracket bonding), and revised the manuscript and gave a final approval for the manuscript to be published. Both authors read and approved the final manuscript.

Received: 1 February 2014 Accepted: 19 March 2014

Published online: 18 July 2014

\section{References}

1. Oesterle $L$, Shellhart WC. Effect of aging on the shear bond strength of orthodontic brackets. Am J Orthod Dentofacial Orthop. 2008; 133:716-20.

2. Tufekci E, Dixon JS, Gunsolley JC, Lindauer SJ. Prevalence of white spot lesions during orthodontic treatment with fixed appliances. Angle Orthod. 2011; 81:206-10.

3. Ogaard B, Rølla G, Arends J, Ten Cate JM. Orthodontic appliances and enamel demineralization. Part 2. Prevention and treatment of lesions. Am J Orthod Dentofacial Orthop. 1988; 94:123-8.

4. Chang HS, Walsh LJ, Freer TJ. Enamel demineralization during orthodontic treatment. Aetiology and prevention. Aust Dent J. 1997; 42:322-7.

5. O'Reilly MM, Featherstone JD. Demineralization and remineralization around orthodontic appliances: an in vivo study. Am J Orthod Dentofacial Orthop. 1987; 92:33-40

6. Geiger AM, Gorelick L, Gwinnett AJ, Griswold PG. The effect of a fluoride program on white spot formation during orthodontic treatment. Am J Orthod Dentofacial Orthop. 1988; 93:29-37.

7. Boyd RL. Two-year longitudinal study of a peroxide-fluoride rinse on decalcification in adolescent orthodontic patients. J Clin Dent. 1992; 3:83-7.

8. Geiger AM, Gorelick L, Gwinnett AJ, Benson BJ. Reducing white spot lesions in orthodontic populations with fluoride rinsing. Am J Orthod Dentofacial Orthop. 1992: 101:403-7.

9. Enaia M, Bock N, Ruf S. White-spot lesions during multibracket appliance treatment: a challenge for clinical excellence. Am J Orthod Dentofacial Orthop. 2011; 140:e17-24.

10. Cook PA. Direct bonding with glass ionomer cement. J Clin Orthod. 1990; 24:509-11.

11. Pascotto RC, Navarro MF, Capelozza Filho L, Cury JA. In vivo effect of a resin-modified glass ionomer cement on enamel demineralization around orthodontic brackets. Am J Orthod Dentofacial Orthop. 2004; 125:36-41.

12. Tabrizi A, Cakirer B. A comparative evaluation of casein phosphopeptideamorphous calcium phosphate and fluoride on the shear bond strength of orthodontic brackets. Eur J Orthod. 2011; 33:282-7.

13. Cehreli SB, Sar C, Polat-Özsoy O, Unver B, Ozsoy S. Effects of a fluoridecontaining casein phosphopeptide-amorphous calcium phosphate complex on the shear bond strength of orthodontic brackets. Eur J Orthod. 2012; 34:193-7.

14. Poosti M, Ramazanzadeh B, Zebarjad M, Javadzadeh P, Naderinasab M, Shakeri MT. Shear bond strength and antibacterial effects of orthodontic composite containing $\mathrm{TiO}_{2}$ nanoparticles. Eur J Orthod. 2013; 35:676-9.

15. Al-Musallam TA, Evans CA, Drummond JL, Matasa C, Wu CD. Antimicrobial properties of an orthodontic adhesive combined with cetylpyridinium chloride. Am J Orthod Dentofacial Orthop. 2006; 129:245-51.

16. Lodaya SD, Keluskar KM, Naik V. Evaluation of demineralization adjacent to orthodontic bracket and bond strength using fluoride-releasing and conventional bonding agents. Indian J Dent Res. 2011; 22:44-9.

17. Pseiner BC, Freudenthaler J, Jonke E, Bantleon HP. Shear bond strength of fluoride-releasing orthodontic bonding and composite materials. Eur J Orthod. 2010; 32:268-73.

18. Yetkiner E, Ozcan M, Wegehaupt FJ, Wiegand A, Eden E, Attin T. Effect of a low-viscosity adhesive resin on the adhesion of metal brackets to enamel etched with hydrochloric or phosphoric acid combined with conventional adhesives. J Adhes Dent. 2013; 15:575-81.

19. Jia L, Stawarczyk B, Schmidlin PR, Attin T, Wiegand A. Effect of caries infiltrant application on shear bond strength of different adhesive systems to sound and demineralized enamel. J Adhes Dent. 2012; 14:569-74.

20. de Souza-e-Silva CM, Parisotto TM, Steiner-Oliveira C, Kamiya RU, Rodrigues LK, Nobre-dos-Santos M. Carbon dioxide laser and bonding materials reduce enamel demineralization around orthodontic brackets. Lasers Med Sci. 2013; 28:111-8.

21. Fekrazad R, Ebrahimpour L. Evaluation of acquired acid resistance of enamel surrounding orthodontic brackets irradiated by laser and fluoride application. Lasers Med Sci. (In press).

22. Manfred L, Covell DA, Crowe JJ, Tufekci E, Mitchell JC. A novel biomimetic orthodontic bonding agent helps prevent white spot lesions adjacent to brackets. Angle Orthod. 2013; 83:97-103.

23. Pithon MM, Ferraz CS, Oliveira GD, Dos Santos AM. Effect of different concentrations of papain gel on orthodontic bracket bonding. Prog Orthod. 2013; 14:22.

24. Chow CK, Wu CD, Evans CA. In vitro properties of orthodontic adhesives with fluoride or amorphous calcium phosphate. Int Dent. 2011; 2011:583521.

25. Schmidlin PR, Sener B, Attin T, Wiegand A. Protection of sound enamel and artificial enamel lesions against demineralization: caries infiltrant versus adhesive. J Dent. 2012; 40:851-6.

26. Montasser MA, Drummond JL. Reliability of the adhesive remnant index score system with different magnification. Angle Orthod. 2009; 79:773-6.

27. Årtun J, Bergland S. Clinical trials with crystal growth conditioning as an alternative to acid-etch enamel pretreatment. Am J Orthod. 1984; 85:333-40.

28. Hayakawa T, Kikutake K, Nemoto K. Influence of self etching primer treatment on the adhesion of resin composite to polished dentin and enamel. Dent Mater. 1998; 14:99-105.

29. Hanning M, Reinhardt KJ, Bott B. Self-etching primer vs phosphoric acid: an alternative concept for composite-to-enamel bonding. Oper Dent. 1999; 24:172-80.

30. Montasser MA, Drummond JL, Roth JR, Al-Turki L, Evans CA. Rebonding of orthodontic brackets. Part II, an XPS and SEM study. Angle Orthod. 2008; 78:537-44.

31. Di Hipólito V, Fernando de Goes M, Rocha de Oliveira Carrilho M, Chan DCN, Daronch M, Sinhoreti MAC. SEM evaluation of contemporary selfetching primers applied to ground and unground enamel. J Adhes Dent 2005; 7:203-11.

32. Pashley DH, Tay FR. Aggressiveness of contemporary self etching adhesives part II: etching effects on unground enamel. Dent Mater. 2001 $17: 430-44$.

33. Van Meerbeek B, De Munck J, Yoshida Y, Inoue S, Vargas M, Vijay P, Van Landuyt K, Lamberchats P, Vanherle G. Buonocore memorial lecture adhesion to enamel and dentin: current status and future challenges. Oper Dent. 2003; 28:215-35

34. Ten Cate JM. In vitro studies of the effects of fluoride on de- and remineralization. J Dent Res. 1990; 69:614-9.

35. Ten Cate JM, Featherstone JDB. Mechanistic aspects of the interactions between fluoride and dental enamel. Crit Rev Oral Biol Med. 1991; 2:283-96.

36. Naidu E, Stawarczyk B, Tawakoli PN, Attin R, Attin T, Wiegand A. Shear bond strength of orthodontic resins after caries infiltrant preconditioning. Angle Orthod. 2013; 83:306-12.

37. Ekizer A, Zorba YO, Uysal T, Ayrikcila S. Effects of demineralizatoninhibition procedures on the bond strength of brackets bonded to demineralized enamel surface. Korean J Orthod. 2012; 42:17-22.

38. Reynolds IR. A review of direct orthodontic bonding. Br J Orthod. 1975; 2:171-8.

39. Eliades T, Brantley WA. The inappropriateness of conventional orthodontic bond strength assessment protocols. Eur J Orthod. 2000; 22:13-23.

40. Miller JR. Commentary basic concepts concerning bracket failure research. Angle Orthod. 1997; 67:167-8.

41. Söderholm K, Mukherjee R, Longmate J. Filler leachability of composites stored in distilled water or artificial saliva. J Dent Res. 1996; 75:1692-9.

42. Lee S-Y, Greener EH, Menis DL. Detection of leached moieties from dental composites in fluids simulating food and saliva. Dent Mater. 1995; 11:348-53.

43. David VA, Staley RN, Bigelow HF, Jakobsen JR. Remnant amount and cleanup for 3 adhesives after debracketing. Am J Orthod Dentofacial Orthop. 2002; 121:291-6. 
44. O'Brien KD, Wattas DC, Read MJF. Residual debris and bond strength - is there a relationship? Am J Orthod Dentofacial Orthop. 1988; 94:222-30.

45. Delport A, Grobler SR. A laboratory evaluation of tensile bond strength of some orthodontic bonding resins to enamel. Am J Orthod Dentofacial Orthop. 1988; 93:133-7.

46. Bishara SE, Vonwald L, Olsen ME, Laffoon JF. Comparisons of two approaches for removing excess adhesive during the bonding procedure. Angle Orthod. 2000; 70:149-53.

doi:10.1186/s40510-014-0034-0

Cite this article as: Montasser and Taha: Effect of enamel protective agents on shear bond strength of orthodontic brackets. Progress in Orthodontics 2014 15:34.

\section{Submit your manuscript to a SpringerOpen ${ }^{\circ}$} journal and benefit from:

- Convenient online submission

- Rigorous peer review

- Immediate publication on acceptance

- Open access: articles freely available online

- High visibility within the field

- Retaining the copyright to your article 